\title{
Oronasal and Tracheostomy Delivery of Soft Mist and Pressurized Metered-Dose Inhalers With Valved Holding Chamber
}

\author{
Ariel Berlinski MD and Brandy Cooper
}

\begin{abstract}
BACKGROUND: Some pediatric tracheostomized patients who receive inhaled drugs undergo decannulation, and it is unknown whether the dose has to be adjusted. Pressurized metereddose inhalers (pMDIs) and soft mist inhalers (SMIs) used with valved holding chambers (VHCs) made of non-electrostatic material are available. We hypothesized that using an SMI and changing the delivery route from tracheostomy to oronasal would increase lung dose. METHODS: Four units of a metallic VHC were studied with albuterol hydrofluoroalkane (pMDI) and albuterol/ipratropium bromide with an SMI using an anatomically correct in vitro model of a 5-y-old spontaneously breathing tracheostomized child. The drug was captured in a filter and was termed lung dose. We tested breathing patterns with tidal volumes of 50,155 , and $300 \mathrm{~mL}$. A mask and a special adapter were used as interfaces for oronasal and tracheostomy delivery, respectively. Spectrophotometry $(276 \mathrm{~nm})$ was used to determine albuterol concentration. RESULTS: The use of SMI resulted in a higher lung dose than the pMDI for all tested conditions except delivery through tracheostomy with tidal volume of $155 \mathrm{~mL}(P=.69)$. Switching from oronasal to tracheostomy delivery increased the lung dose for all tested conditions except for the pMDI with the $300-\mathrm{mL}$ tidal volume $(P=.83)$. The use of SMI resulted in higher deposition in the tracheostomy tube than the pMDI. CONCLUSIONS: In general, an SMI delivers a higher lung dose than a pMDI when using a metallic spacer during oronasal and tracheostomy route with the latter providing a higher lung dose. Key words: tracheostomy; drug delivery; aerosol; valved holding chamber; metered dose inhaler; artificial airway; soft mist inhaler; Respimat; pediatrics. [Respir Care 2016;61(7):913-919. (C) 2016 Daedalus Enterprises]
\end{abstract}

\section{Introduction}

Many pediatric patients who undergo tracheostomy receive long-term treatment with inhaled aerosols. ${ }^{1}$ Also,

\footnotetext{
Dr Berlinski is affiliated with the Pulmonology Section, Department of Pediatrics, University of Arkansas for Medical Sciences and the Pediatric Aerosol Research Laboratory, Arkansas Children's Hospital Research Institute, Little Rock, Arkansas. Ms Cooper is affiliated with the College of Pharmacy, University of Arkansas for Medical Sciences, Little Rock, Arkansas.
}

Ms Cooper was supported by a University of Arkansas for Medical Sciences College of Pharmacy Summer Research Fellowship. The Pediatric Aerosol Research Laboratory at Arkansas Children's Hospital Research Institute was partially established by and receives partial support from the George Endowment for Asthma. Dr Berlinski has disclosed relationships with Vertex, AbbVie, Aptalis Pharma, Genentech, Janssen Research and Development, Gilead, Teva, Philips, Novartis, the National Institutes of Health, and the Therapeutic Development Network. Ms Cooper has disclosed no conflicts of interest. many children with chronic lung disease and tracheostomy could be decannulated, but they continue to require inhaled aerosols. Currently, there are no guidelines to help the practitioner optimize drug delivery in that patient population. $^{2}$

A survey of aerosol delivery practices in tracheostomized children revealed that $92 \%$ of the responders used pressurized metered-dose inhalers (pMDIs). ${ }^{1}$ In addition, $83 \%$ of those using unassisted methods reported using valved holding chambers (VHCs). The goal of using a VHC is to remove the need of actuation and inhalation coordination. ${ }^{3}$ There are several components that are crucial to optimal

\footnotetext{
Correspondence: Ariel Berlinski MD, Pediatric Pulmonology Section, 1 Children's Way, Slot 512-17, Little Rock, AR 72202. E-mail: BerlinskiAriel@uams.edu.
}

DOI: $10.4187 /$ respcare. 04575 
VHC performance (ie, material used). ${ }^{3}$ There are 2 types of non-electrostatic materials used for VHCs: polymer and metal. Previous studies have reported different performance among VHCs made of different non-electrostatic materials. $^{4}$

Traditionally, metered-dose inhalers contained propellants, but more recently, a new type of inhaler has been introduced in the market. The Respimat (Boehringer Ingelheim Pharmaceuticals, Ridgefield, New Jersey), also known as the Soft Mist inhaler (SMI) is a propellant-free metered-dose inhaler that provides a slower mist than traditional pMDIs. ${ }^{5}$ The delivery device is approved in the United States and in Europe for the following drugs: albuterol/ipratropium bromide, tiotropium bromide/olodaterol, olodaterol, and tiotropium bromide. The pMDI for albuterol/ipratropium bromide was replaced by an SMI in the United States and is no longer available. The use of a $\mathrm{VHC}$ is not required in adults and older children. ${ }^{6,7}$ However, handling studies of the Respimat device recommend the addition of a VHC in children $\leq 5$ y old. ${ }^{8}$ The Respimat with a VHC effectively delivered aerosol to a group of sleeping infants. ${ }^{9}$

Due to the paucity of clinical studies, most of our insight on optimization of aerosol delivery with pMDIs and VHCs through tracheostomy derives from in vitro studies. ${ }^{10-12}$ Those studies reported that the type of VHC, breathing patterns, and other factors affect drug delivery of pMDI through tracheostomies. Recently, Cooper et al ${ }^{12}$ compared oronasal and tracheostomy delivery of albuterol using a pMDI with 2 different VHCs and a continuous output jet nebulizer. They found that the type of VHC and breathing pattern affect the difference between the lung dose obtained via tracheostomy and oronasal routes. ${ }^{12}$

Knowledge regarding the effect of different drug delivery devices (pMDI vs SMI) and VHCs (metallic vs antistatic polymer) is needed. We hypothesize that the use of SMI, larger tidal volumes, and changing the delivery route from tracheostomy to oronasal will result in a higher lung dose. We also hypothesize that a metallic VHC will perform similarly to one of similar size made of antistatic polymer.

\section{Methods}

The experiments were performed at the Pediatric Aerosol Research Laboratory at Arkansas Children's Research Institute, Little Rock, Arkansas.

\section{Breathing Model}

A previously published anatomically correct model of a spontaneously breathing tracheostomized 5-year-old child was used. ${ }^{12}$ Briefly, a 3-dimensional printed oronasal head

\section{QUICK LOOK}

\section{Current knowledge}

Spontaneously breathing tracheostomized pediatric patients often receive inhaled treatments with metereddose inhalers and valved holding chambers. Soft mist inhalers are currently available in the United States.

\section{What this paper contributes to our knowledge}

A soft mist inhaler delivered more albuterol than a metered-dose inhaler when both were used with a non-electrostatic chamber in an anatomically correct model of a spontaneously breathing tracheostomized child.

model of a 5-y-old child was connected in series to a low dead space filter holder and a breathing simulator (PARI Compass, Munich, Germany) (Fig. 1). The breathing patterns of an infant (tidal volume $50 \mathrm{~mL}$, breathing frequency 30 breaths/min, inspiratory-expiratory ratio 1:3), a child (tidal volume $155 \mathrm{~mL}$, breathing frequency 25 breaths $/ \mathrm{min}$, inspiratory-expiratory ratio 1:2), and an older child (tidal volume $300 \mathrm{~mL}$, breathing frequency 25 breaths $/ \mathrm{min}$, inspiratory-expiratory ratio $1: 2$ ) were used. The model had a hole to allow the insertion of the tracheostomy tube that was occluded during oronasal delivery experiments. A 4.5- $\mathrm{mm} / 6.3-\mathrm{mm}$ inner diameter/outer diameter tracheostomy tube (TRACHOE Medical GmbH, Nieder-Olm, Germany) was used.

\section{Devices}

Albuterol pMDI (Ventolin hydrofluoroalkane [HFA], $90 \mu \mathrm{g} /$ actuation; GlaxoSmithKline, Brentford, United Kingdom) and SMI albuterol/ipratropium bromide (Combivent Respimat, $100 \mu \mathrm{g}$ of albuterol/actuation, Boehringer Ingelheim Pharmaceuticals, Ridgefield, New Jersey) were used. During oronasal delivery, 4 units of metallic VHC (Vortex Tracheo, PARI GmbH, Starnberg, Germany) with its medium size mask (Felix Frog SmartTouch Mask, PARI Midlothian, Virginia) were used (Fig. 2). The Vortex Tracheo is commercialized in Europe but not in the United States. The metallic VHC is not transparent and has a volume of $194 \mathrm{~mL}$. The operation of the inhalation valve can be observed through the mouthpiece. During tracheostomy delivery, the VHC was connected to the tracheostomy tube with a special adapter that is present in the Vortex Tracheo, and during oronasal delivery, the adapter was removed and the mask was connected.

\section{Protocol}

Oronasal Delivery. A new disposable filter (PARI Respiratory Equipment, Midlothian, Virginia) was used 


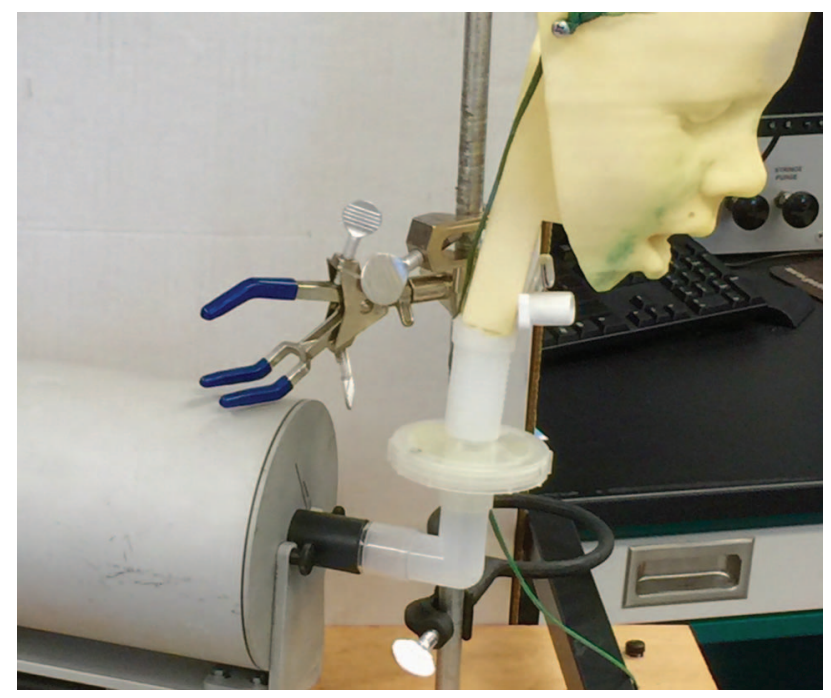

Fig. 1. Experimental setup.

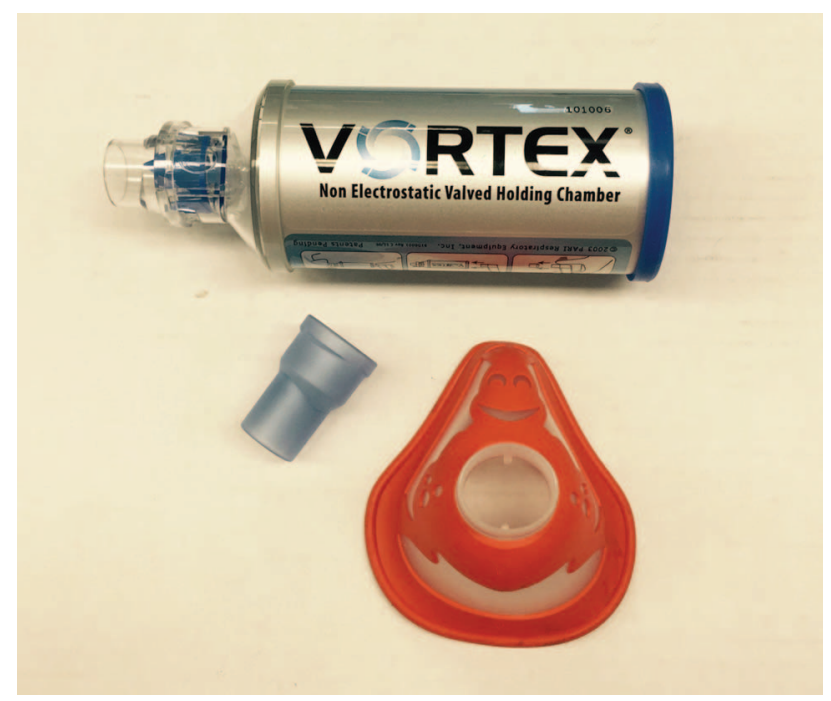

Fig. 2. Devices and interfaces used during oronasal and tracheostomy aerosol delivery. Top: Vortex valved holding chamber. Middle: Tracheostomy adapter. Bottom: Face mask.

with each run. The model was operated in the vertical position as previously reported. ${ }^{11,12}$ After selecting a breathing pattern, the accuracy of the tidal volume was verified using a mass flow meter (TSI, Shoreview, Minnesota). ${ }^{11,12}$ The seal between the head/airway model and the mask was achieved with putty. ${ }^{12}$ The pMDI was primed with 4 puffs at the beginning of every testing day and was shaken for $5 \mathrm{~s}$ before each actuation. The SMI was primed before use as per the manufacturer's recommendations. The same canister was used for each run to minimize variability. The pMDI and the SMI were inserted into the back of the VHC. The breathing simulator provided 7 breaths, and the inhalers were actuated at the beginning of the second inhalation. ${ }^{12} \mathrm{~A}$ total of 10 actuations were utilized to improve drug recovery, and the used filter was processed for albuterol concentration. ${ }^{11,12}$ The VHCs were cleaned and air-dried, and lack of residual drug was verified by spectrophotometry. This process was done for each of the 2 devices (pMDI and SMI) and for each of the 3 breathing patterns (infant, child, and older child).

Tracheostomy Delivery. A similar procedure was used for tracheostomy delivery except that the mask was replaced by a special adapter to connect to the tracheostomy tube. Upon completion of each experiment, the tracheostomy tube was removed and flushed with $10 \mathrm{~mL}$ of double-deionized water. The filter and tracheostomy samples were analyzed with a spectrophotometer.

\section{Albuterol Determination}

After washing with double-deionized water the filter holder and the filter, the latter was placed in a $50-\mathrm{mL}$ tube and was vigorously vortexed and shaken. The samples were analyzed with spectrophotometry at $276 \mathrm{~nm}$ (Biomate 3 UV-visible spectrophotometer, Thermo Fisher Scientific, Waltham, Massachusetts). ${ }^{12}$

\section{Statistical Analysis}

Lung dose (amount of albuterol recovered from the filter) was the main outcome. The comparison of lung doses between oronasal and tracheostomy delivery and between the 2 devices was done with unpaired $t$ test. The effects of breathing patterns for each device/delivery route scenario on lung dose were studied with analysis of variance followed by Tukey test (multiple comparisons). $P$ values of $<.05$ were considered statistically significant. Calculations were done with a statistical software package (Kaleidagraph 4.1, Synergy Software, Reading, Pennsylvania).

\section{Results}

Results are expressed in Table 1 and Figure 3.

\section{Comparison Between pMDI and SMI}

When albuterol with a metallic VHC was delivered through the oronasal route, the SMI achieved higher lung doses than the pMDI. The difference was statistically significant for all breathing patterns but was the largest for the 50-mL tidal volume (8.5-fold).

When albuterol with a metallic VHC was delivered through the tracheostomy tube, the SMI achieved higher lung doses than the pMDI for the breathing patterns with 
Table 1. Lung Dose ( $\mu \mathrm{g}$ of Albuterol) Achieved With Different Valved Holding Chambers With Pressurized Metered-Dose and Soft Mist Inhalers During Oronasal and Tracheostomy Delivery

\begin{tabular}{|c|c|c|c|c|c|c|c|c|c|c|c|c|}
\hline \multirow{2}{*}{$\begin{array}{c}\text { Tidal } \\
\text { Volume, } \mathrm{mL}\end{array}$} & \multicolumn{4}{|c|}{ Oronasal Delivery, $\mu \mathrm{g}$} & \multicolumn{4}{|c|}{ Tracheostomy Delivery, $\mu \mathrm{g}$} & \multicolumn{4}{|c|}{$\begin{array}{l}\text { Tracheostomy/Oronasal } \\
\text { Delivery, } \mu \mathrm{g}\end{array}$} \\
\hline & pMDI & SMI & $P^{*}$ & SMI/pMDI & pMDI & SMI & $P^{*}$ & SMI/pMDI & pMDI & $P \dagger$ & SMI & $P \dagger$ \\
\hline 50 & $11.7 \pm 3.8$ & $99.4 \pm 7.1$ & $<.001$ & 8.5 & $23.7 \pm 5$ & $71.4 \pm 12.9$ & .003 & 3 & 2 & .009 & 0.7 & .01 \\
\hline 155 & $89.7 \pm 7.6$ & $179.2 \pm 4.3$ & $<.001$ & 2 & $223.1 \pm 52.9$ & $214.8 \pm 14.2$ & .69 & 1 & 2.5 & $<.001$ & 1.2 & .01 \\
\hline 300 & $173.6 \pm 9$ & $211.1 \pm 7.5$ & $<.001$ & 1.2 & $175.9 \pm 26.6$ & $314.1 \pm 34.4$ & $<.001$ & 1.8 & 1 & .83 & 1.5 & .007 \\
\hline \multicolumn{13}{|c|}{$\begin{array}{l}\text { * Comparison between lung dose obtained with pressurized metered-dose and soft mist inhaler. } \\
\dagger \text { Comparison between lung dose obtained via tracheostomy and oronasal delivery. } \\
\text { pMDI = pressurized metered-dose inhaler } \\
\text { SMI = soft mist inhaler }\end{array}$} \\
\hline
\end{tabular}

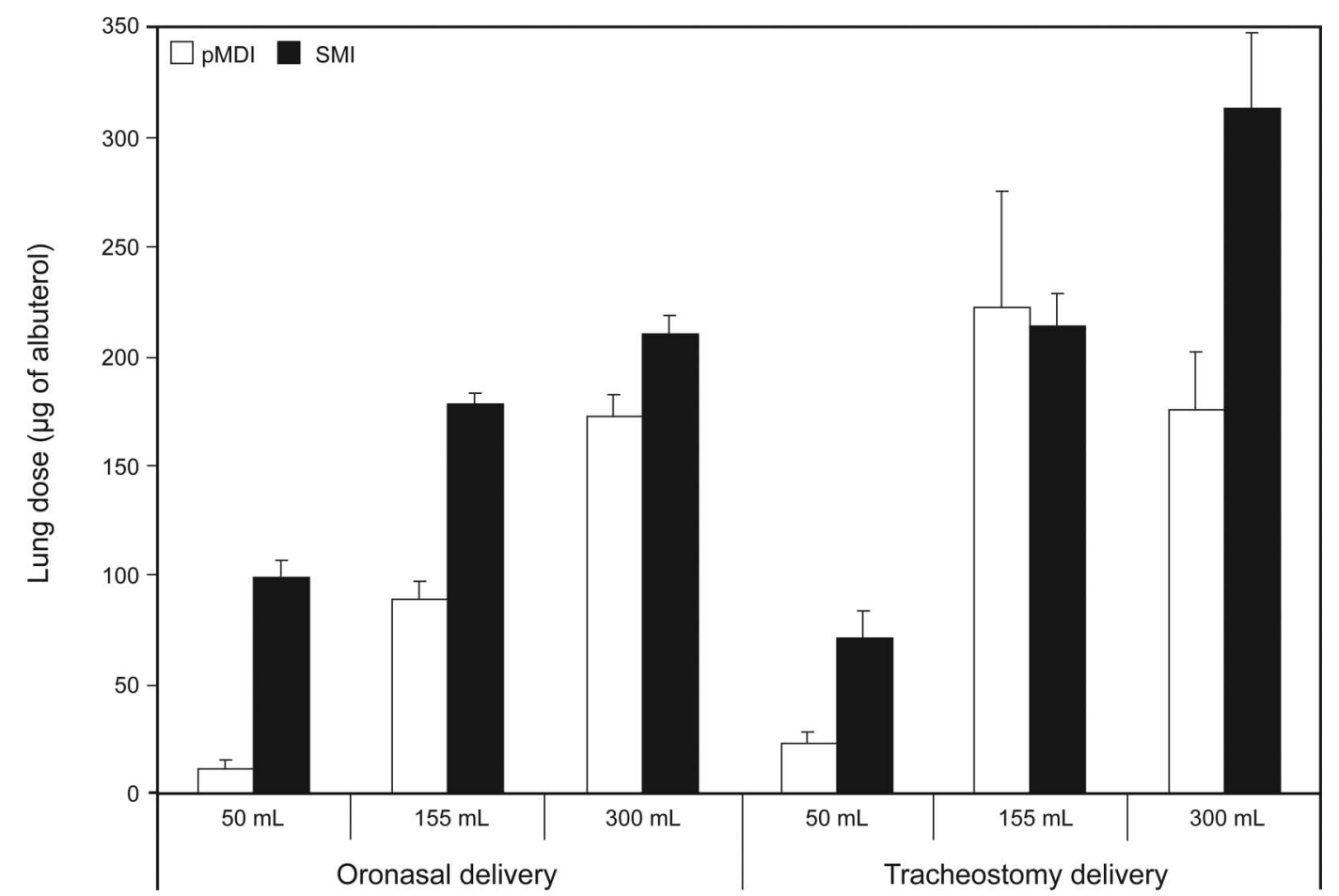

Fig. 3. Lung dose achieved with a metallic valved-holding chamber with pressurized metered-dose inhaler (pMDI) and soft mist inhaler (SMI) with different breathing patterns during oronasal and tracheostomy albuterol delivery.

a 50-mL (3-fold difference) and 300-mL (1.8-fold difference) tidal volume. However, no difference was noted for the breathing pattern with a tidal volume of $155 \mathrm{~mL}$ $(P=.69)$.

\section{Comparison Between Oronasal and Tracheostomy Delivery}

When the delivery route of albuterol administered with pMDI and metallic VHC was changed from tracheostomy to oronasal, lung dose increased for the breathing patterns with 50 - and $155-\mathrm{mL}$ tidal volumes but not for the breathing patterns with a tidal volume of $300 \mathrm{~mL}(P=.83)$. The tracheostomy/oronasal lung dose ratio for the pMDI with metallic VHC was 2, 2.5, and 1 for breathing patterns with tidal volumes of 50,155, and $300 \mathrm{~mL}$, respectively.

When the delivery route of albuterol administered with SMI and metallic VHC was changed from tracheostomy to oronasal, lung dose decreased for the breathing pattern with tidal volume of $50 \mathrm{~mL}$ but increased for the other. The tracheostomy/oronasal lung dose ratio for the SMI with metallic VHC was $0.7,1.2$, and 1.5 for breathing patterns with tidal volumes of 50,155 , and $300 \mathrm{~mL}$, respectively. 


\section{Breathing Patterns}

When using a pMDI with a metallic VHC through the oronasal route, the lung dose was higher for higher tidal volumes $(P=.001)$. During delivery through tracheostomy, the breathing pattern with a $50-\mathrm{mL}$ tidal volume was lower than others $(P<.001)$. The lung dose achieved with breathing patterns with tidal volumes of 155 and $300 \mathrm{~mL}$ were similar $(P=.059)$. When using an SMI with a metallic VHC through the oronasal and tracheostomy routes, the lung dose increased with higher tidal volumes $(P<.001)$.

\section{Amount of Drug Deposited in the Tracheostomy Tube}

The amount of albuterol retained in the tracheostomy tube when using a pMDI and Vortex was $0.7,3$, and $12.1 \%$ of the nominal dose for breathing patterns with tidal volumes of 50,155 , and $300 \mathrm{~mL}$, respectively. The tidal volume of $300 \mathrm{~mL}$ had the highest drug retention $(P<.001)$.

The amount of albuterol retained in the tracheostomy tube when using an SMI and metallic VHC increased with higher tidal volumes $(P<.001)$ and was $1.3,16.1$, and $36.5 \%$ of the nominal dose for breathing patterns with tidal volumes of 50, 155, and $300 \mathrm{~mL}$, respectively. The SMI had a higher intra-tracheostomy deposition than the pMDI for all breathing patterns $(P=.009, P=.001$, and $P=.004$ for the breathing patterns with a tidal volume of 50, 155, and $300 \mathrm{~mL}$ respectively.

\section{Discussion}

We found that in general, albuterol delivery via an oronasal and tracheostomy route using a metallic VHC resulted in a higher lung dose with an SMI than with a pMDI. We also found that in general, the lung dose was higher for tracheostomy than for oronasal delivery for both pMDI and SMI devices used with a metallic VHC. Finally, deposition in the tracheostomy tube was higher for SMI than pMDI when both were used with a metallic VHC.

The finding that the use of an SMI provided a larger lung dose than the pMDI is consistent with the characteristics of the aerosols produced by both inhalers. ${ }^{5}$ The SMI produces an aerosol that is 10 times slower and is ejected for a 3 -fold greater duration than the one produced by the pMDI. ${ }^{5}$ We speculate that the difference in magnitude noted among different tidal volumes could be explained by the relative influence of difference in speed and duration of the aerosols. We speculate that a slower aerosol will result in less impaction and will be mainly responsible for the increase in lung dose found with tidal volumes of 155 and $300 \mathrm{~mL}$. We speculate that the higher magnitude of the increase in lung dose noted with the $50-\mathrm{mL}$ tidal volume is heavily influenced by the duration of aerosol because it is only one quarter of the volume of the VHC compared with 75 and $100 \%$ of the volume for the tidal volumes of 155 and $300 \mathrm{~mL}$, respectively. Our findings are also consistent with adult studies comparing lung deposition between a pMDI and an SMI but without the use of a VHC. ${ }^{13}$ Wachtel et al ${ }^{14}$ reported a lung dose of $33 \%$ for an SMI using a different airway model but with a similar VHC (Vortex). The difference with our lung dose $(21.1 \%)$ could be due to the fact that we used a different airway model and breathing pattern. In another study, Amirav et al ${ }^{15}$ reported a 30 and $60 \%$ lung dose in a 20-monthold model using breathing patterns with a tidal volume of 80 and $120 \mathrm{~mL}$, respectively. They also reported a 20 and $40 \%$ lung dose in a 14-month-old model using breathing patterns with a tidal volume of 50 and $80 \mathrm{~mL}$, respectively. ${ }^{15}$ The same group reported a $4.2 \%$ lung deposition in a group of 9-month-old infants using a VHC and Respimat. ${ }^{9}$ Their in vitro model overestimated lung deposition by a 4 -fold difference. ${ }^{9,15}$ Our model yielded a $9 \%$ deposition for the breathing pattern with a tidal volume of $155 \mathrm{~mL}$. Again, differences in airway models, breathing patterns, VHCs, and masks could potentially explain these differences.

The finding that during oronasal delivery of pMDI lung dose increased with increasing tidal volumes is consistent with previous reports using anatomically correct and nonanatomically correct models. ${ }^{11,12,16}$ During tracheostomy delivery, lung dose was lower for the $50-\mathrm{mL}$ tidal volume but was similar between the breathing patterns with 155 and $300 \mathrm{~mL}$. This finding is in agreement and disagreement with previous reports. ${ }^{11,12}$ Cooper et al, ${ }^{12}$ using the same model and 2 VHCs made of a nonelectrostatic polymer, reported a direct correlation between tidal volume and lung dose for one (Aerotrach Plus) and a lower lung dose for the 50-mL tidal volume than for other tidal volumes $(155$ and $300 \mathrm{~mL}$ ) with the other VHC (Aerochamber Mini). Berlinski et al, ${ }^{11}$ using a non-anatomically correct model, reported similar findings for the same tracheostomy size and similar breathing patterns (tidal volumes of 80, 155, and $310 \mathrm{~mL}$ ) for the Aerochamber Mini. However, lung dose was similar among all breathing patterns for the Aerotrach. These differences could be explained in part by the characteristics of the models used. Whereas this study and the one by Cooper et al ${ }^{12}$ used a 2-compartment model with bidirectional flow, the study by Berlinski et al $^{11}$ used a 2-compartment model with unidirectional flow. Their model placed a one-way valve at the vocal cord level that opened during expiration in contrast to the bidirectional flow model that allowed air flow through the vocal cords during inspiration and expiration. ${ }^{11,12}$ These findings underscore the fact that data for 
a specific device should not be extrapolated to others and that choice of model could influence results.

Consistent with a previous report, ${ }^{12}$ the amount of drug deposited in the tube increased with increasing tidal volumes. This was true for both the SMI and the pMDI, with the latter being lower than previously reported.12 However, this was in contrast to findings by Berlinski et al ${ }^{11}$ that reported for the pMDI similar deposition for different tidal volumes. The difference could be explained in part by the difference in model (unidirectional vs bidirectional flow) and pMDI formulation (Proair HFA vs Ventolin HFA). ${ }^{11,17}$ An unexpected finding was that deposition in the tracheostomy tube was higher with the SMI than with the pMDI. We hypothesized that the lower speed would have resulted in lower impaction in the tube. We speculate that the longer duration of the aerosol of the SMI allowed more time to deposit in the tube.

This study adds to the body of knowledge provided by previously reported data using the same model and with experiments done by the same operator and allows a direct comparison with fewer biases than are usually found. ${ }^{12}$ During oronasal delivery of pMDI with tidal volumes of 50 and $155 \mathrm{~mL}$, the Aerochamber Mini was better than the others and the Aerotrach VHC was better than the Vortex VHC. However, when the large tidal volume $(300 \mathrm{~mL})$ was used, lung dose was higher for the Aerochamber VHC followed by the Vortex VHC and then the Aerochamber Mini VHC. During tracheostomy delivery of pMDI, the Vortex VHC performed similarly to the Aerotrach VHC for the lower tidal volume $(50 \mathrm{~mL})$, and both had a lower lung dose than the Aerochamber Mini VHC. However, when larger tidal volumes were used $(155$ and $300 \mathrm{~mL})$, the Vortex VHC outperformed the other 2. These data suggest the existence of a complex relationship between lung dose obtained with a pMDI, the breathing pattern, and the type of VHC used.

Our findings have several clinical implications: (1) pMDI and SMI are not interchangeable, and the magnitude of the difference in drug delivery varies significantly with different tidal volumes; and 2) recommendations based on data obtained with a specific device/formulation should not be assumed to be equal to other combinations until proven. In the absence of dosing equivalency, clinicians should consider starting at a lower dose and titrating to effect when switching from a less efficient to a more efficient delivery system. Given the number of variables that play a role in drug delivery (VHCs, masks, aerosol generators, breathing patterns, etc), practitioners should consider utilizing products that are cost-efficient and have been studied in conditions similar to the ones in which they will be used.

The major limitation of this study is its in vitro nature, which does not provide the biological variability that occurs during real life. These methods tend to overestimate lung dose, especially the fraction that is smaller than the $0.5 \mu \mathrm{m}$ that would have been exhaled but is trapped in the filter instead. Nonetheless, these are well-established methods in aerosol research. The findings of our study should not be extrapolated to other devices, tracheostomy size tubes, and breathing patterns. Another limitation is that we did not measure particle size of the aerosols that left the tip of the tracheostomy tube.

\section{Conclusions}

The experiments reported in this study should caution practitioners from interchangeably using pMDI and SMI inhalers. Our findings underscore that data obtained with one device/formulation combination cannot be extrapolated to others. Whereas a dose increase might be necessary for the patients with low tidal volume when changing the delivery route of an SMI from an oronasal to a tracheostomy delivery route, the opposite action should be taken for larger tidal volumes and when an SMI is used. Animal and human studies are needed to help improve in vitro/in vivo correlation.

\section{REFERENCES}

1. Willis LD, Berlinski A. Survey of aerosol delivery techniques to spontaneously breathing tracheostomized children. Respir Care 2012; 57(8):1234-1241.

2. Amirav I, Newhouse MT. Aerosol therapy in tracheotomized children: time for guidelines! Respir Care 2012;57(8):1350.

3. Rubin BK. Air and soul: the science and application of respiratory therapy. Respir Care 2010;55(7):911-921.

4. Rau JL, Coppolo DP, Nagel MW, Avvakoumova VI, Doyle CC, Wiersema KJ, Mitchell JP. The importance of nonelectrostatic materials in holding chambers for delivery of hydrofluoralkane albuterol. Respir Care 2006;51(5):503-510.

5. Hochrainer D, Hölz H, Kreher C, Scaffidi L, Spallek M, Wachtel H. Comparison of the aerosol velocity and spray duration of Respimat soft mist inhalers and pressurized metered dose inhalers. J Aerosol Med 2005;18(3):273-282.

6. Anderson P. Use of Respimat ${ }^{\circledR}$ Soft Mist ${ }^{\mathrm{TM}}$ inhaler in COPD patients. Int J Chron Obstruct Pulmon Dis 2006;1(3):251-259.

7. von Berg A, Jeena PM, Soemantri PA, Vertruyen A, Schmidt P, Gerken F, Razzouk H. Efficacy and safety of ipratropium bromide plus fenoterol inhaled via Respimat ${ }^{\circledR}$ Soft Mist ${ }^{\mathrm{TM}}$ inhaler vs. a conventional metered dose inhaler plus spacer in children with asthma. Pediatr Pulmonol 2004;37(3):264-272.

8. Kamin W, Frank M, Kattenbeck S, Moroni-Zentgraf P, Wachtel H, Zielen S. A handling study to assess use of Respimat ${ }^{\circledR}$ Soft Mist ${ }^{\mathrm{TM}}$ inhaler in children under 5 years old. J Aerosol Med Pulm Drug Deliv 2015;28(5):372-381.

9. Amirav I, Newhouse MT, Luder A, Halamish A, Omar H, Gorenberg M. Feasibility of aerosol drug delivery to sleeping infants: a prospective observational study. BMJ Open 2014;4(3):e004124.

10. Piccuito CM, Hess DR. Albuterol delivery via tracheostomy tube. Respir Care 2005;50(8):1071-1076.

11. Berlinski A, Chavez A. Albuterol delivery via metered dose inhaler in a spontaneously breathing pediatric tracheostomy model. Pediatr Pulmonol 2013;48(10):1026-1034. 
12. Cooper B, Berlinski A. Albuterol delivery via facial and tracheostomy route in a model of a spontaneously breathing child. Respir Care 2015;60(12):1749-1758.

13. Brand P, Hederer B, Austen G, Dewberry H, Meyer T. Higher lung deposition with Respimat Soft Mist inhaler than HFA-MDI in COPD patients with poor technique. Int J Chron Obstruct Pulmon Dis 2008; 3(4):763-770.

14. Wachtel H, Bickman Deborah, Breitkreutz J, Langguth P. Can pediatric throat models and air flow profiles improve our dose finding strategy? Respir Drug Deliv 2010;1:195-204.
15. Amirav I, Borojeni AA, Halamish A, Newhouse MT, Golshahi L. Nasal versus oral aerosol delivery to the "lungs" in infants and toddlers. Pediatr Pulmonol. 2014. doi: 10.1002/ppul.22999.

16. Chavez A, McCracken A, Berlinski A. Effect of face mask static dead volume, respiratory rate and tidal volume on inhaled albuterol delivery. Pediatr Pulmonol 2010;45(3):224-229.

17. Hautmann J, Godoy SE, Marshik P, Chand R, McConville J, Krishna $S$ et al. Effect of time between actuation on the dose variability for three metered dose inhalers. Respir Drug Deliv Eur 2013;2: 429-434.

This article is approved for Continuing Respiratory Care Education credit. For information and to obtain your CRCE

(free to AARC members) visit 